\title{
Instanton Rate Constant Calculations Close to and Above the Crossover Temperature
}

\author{
Sean McConnell*, Johannes Kästner*
}

\begin{abstract}
Canonical instanton theory is known to overestimate the rate constant close to a system-dependent crossover temperature and is inapplicable above that temperature. We compare the accuracy of the reaction rate constants calculated using recent semiclassical rate expressions to those from canonical instanton theory. We show that rate constants calculated purely from solving the stability matrix for the action in degrees of freedom orthogonal to the instanton path is not applicable at arbitrarily low temperatures and employ two methods to overcome this. Furthermore, as a by-product of the developed methods, we derive a simple correction to canonical instanton theory that can alleviate this known overestimation of rate constants close to the crossover temperature. The combined methods accurately reproduce the rate constants of the canonical theory along the whole temperature range without the spurious overestimation near the crossover temperature. We calculate and compare rate constants on three different reactions: $\mathrm{H}$ in the Müller-Brown potential, methylhydroxycarbene $\rightarrow$ acetaldehyde and $\mathrm{H}_{2}+\mathrm{OH} \rightarrow \mathrm{H}+\mathrm{H}_{2} \mathrm{O}$.
\end{abstract}

Keywords: Atom tunneling, reaction rate, instanton theory, low-temperature reactivity, computational chemistry, software update

\footnotetext{
*Institute for Theoretical Chemistry, University of Stuttgart, Pfaffenwaldring 55, 70569 Stuttgart, Germany
} 


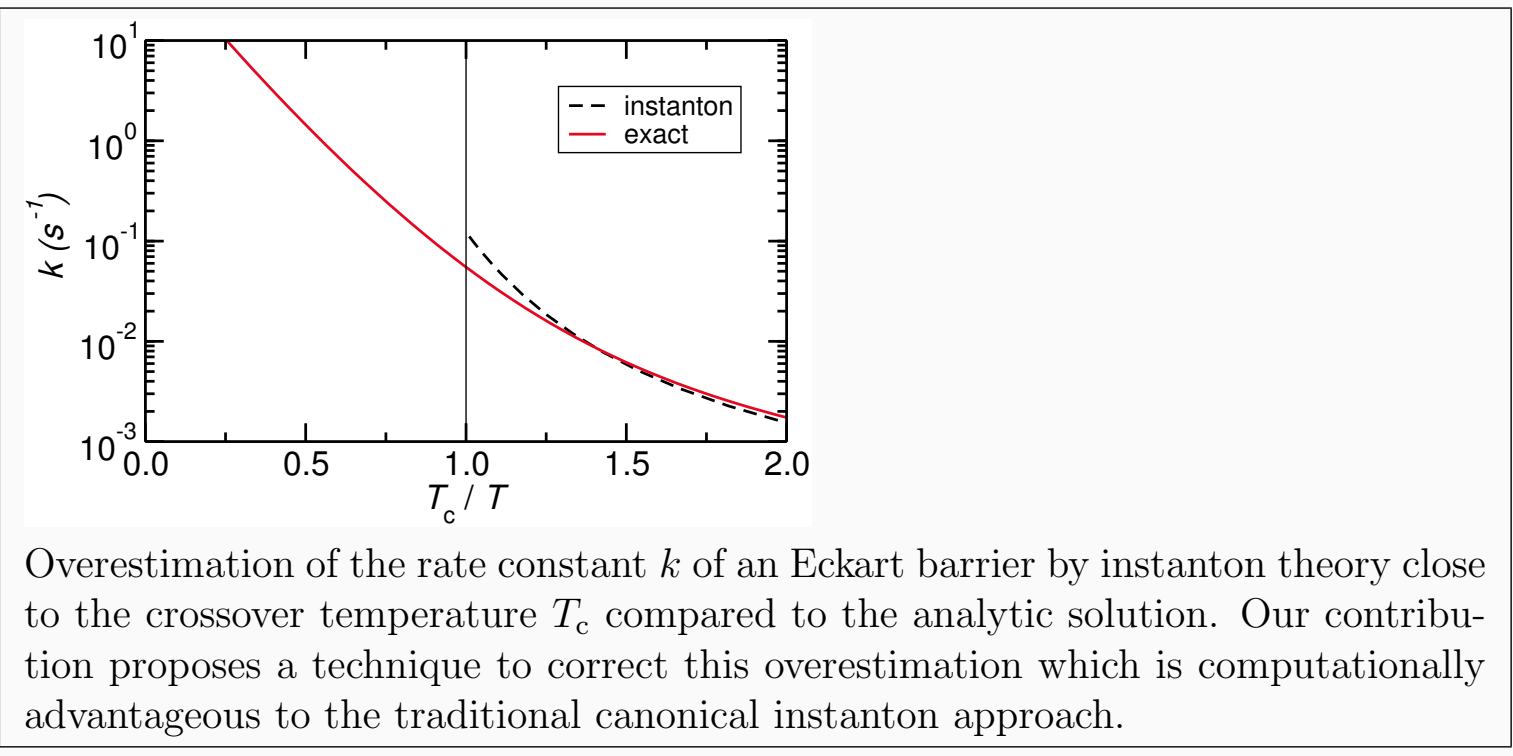




\section{Introduction}

Semiclassical instanton theory is a well-established technique to calculate reaction rate constants including quantum tunneling of atoms. $\frac{112}{12}$ In its most common formulation, it is in-

applicable above a system-dependent crossover temperature $T_{\mathrm{c}}=\frac{\hbar\left|\omega_{b}\right|}{2 \pi k_{\mathrm{B}}}$ and is known to overestimate rate constants close to, but below $T_{c}$. Here $\left|\omega_{b}\right|$ is the absolute value of the imaginary frequency at the transition state, and $k_{\mathrm{B}}$ is the Boltzmann constant. Moreover, in terms of the computational demands, it requires the diagonalisation of a matrix of dimension $N P$, where $N$ is three times the number of atoms in the reaction and $P$ is the number of control points (images) of the instanton.

Recently, a new formulation of instanton theory has been postulated ${ }^{3} \sqrt[5]{5}$, which, with our modifications, can overcome each of these problems while retaining good agreement with the canonical theory.

This implementation of instanton theory sits among others based on either evaluating the imaginary part of the free energy or representing the rate in terms of a flux through a surface. $\frac{6-23}{23}$ The evaluation of free energies as a pathway to obtaining rate constants can be traced back to the work in non-hermitian quantum mechanics in predicting the decay of distinct quantum states by Gamow. ${ }^{24}$ The utility of these $\operatorname{Im}(F) /$ flux based methods in accounting for tunnelling lies in their applicability to systems with many degrees of freedom. Though more rigorous techniques exist for obtaining rate constants, e.g. wave-packet dynamics, $\frac{25}{25}$ these approaches are computationally prohibitive for all but the simplest of systems, besides which, a number of publications exist justifying the agreement between instanton theory and more computationally intensive methods, a selection of which we cite here. $\frac{26 \mid 27}{6}$

We set out to compare the temperature dependent rate constants determined by this new theory, with our modifications, to that of canonical instanton theory. ${ }^{22}$ Our comparison involves 3 different systems: the Müller-Brown potential, the unimolecular reaction of methylhydroxycarbene to acetaldehyde and the bimolecular, gas-phase reaction of a hydrogen molecule with a hydroxyl radical forming water and a hydrogen atom. In terms of the theoretical approach, the main difference between canonical instanton theory and this new formulation ${ }^{3-5}$ lies in the calculation of the instanton partition function and the treatment of 
the Euclidean action therein. Where in canonical instanton theory the temporal integration in the action is discretised and the partition function approximated using Laplace's method, the theory used here splits the action into classical and orthogonal fluctuations. The contribution to the partition function due to these orthogonal fluctuations can be calculated by determining the eigenvalues of a monodromy matrix. The primary motivation behind the chosen test reactions is to gain an appreciation for the efficacy of the method on higher dimensional systems. In higher dimensions, each mode of the eigenvalue spectrum of the monodromy matrix is not so clearly separable from the remaining modes and moreover, a naive approach to obtaining the eigenvalue spectrum may imply an instable instanton orbit. From a theoretical standpoint, these are not necessarily questions of great importance, yet in practical terms they have the potential to limit the adoption of these techniques by a broader community.

The recent formulation ${ }^{3} \sqrt{5}$ is applicable over the full temperature range, above and below the crossover temperature $T_{\mathrm{c}}$. The well known problem $\frac{28 \mid 29}{26}$ of canonical instanton theory, that reaction rate constants are overestimated at temperatures close to, but less than $T_{\mathrm{c}}$, is also solved. Of course, the applicability of instanton theory can also be extended above $T_{\mathrm{c}}$ by switching to a microcanonic expression.

In comparison to canonical instanton theory, another advantage of this recent formulation $^{3-5}$ is a reduced computational burden.

At no stage in the calculation presented here is it necessary to diagonalise a matrix with dimension greater than $N$. This ensures an acceleration in the calculation of $\mathcal{O}\left(P^{2}\right)$ over canonical instanton theory, for this reason, we will refer to this approach henceforth as reduced instanton theory.

Apart from the objective of method comparison, we seek to provide further insights on the formulation and implementation of the theory. To that end, we will show empirically that contributions to the rate constant from tunnelling orthogonal to the instanton path cannot be reliably accounted for by usage of a stability matrix ${ }^{33}$ at arbitrarily low temperatures. We illustrate a few techniques which can circumvent this problem.

The paper is structured as follows. In section 2 we start from the established rate equation and illustrate the features contained in our implementation. Unique to our approach is the 
determination of the rate of change of the tunnelling energy $E_{b}$, and some technical detail in accounting for oscillations orthogonal to the instanton path. Thereafter, in section 3 , we compare the performance of the reduced instanton theory to canonical instanton theory in the aforementioned test cases at temperatures below $T_{\mathrm{c}}$, this is the main objective of this contribution. For rate constants above $T_{\mathrm{c}}$, a comparison to canonical instanton theory is not possible, in this region we therefore compare to the classical theory and to the Bell and Eckart approximations. We discuss the results, potential issues and future directions in sections 4 and 5. All equations are in atomic units $\left(\hbar=m_{e}=4 \pi \epsilon_{0}=1, c=1 / \alpha\right)$. The theory as implemented here is available in the latest update to the quantum chemistry program DL-FIND. 34

\section{Theory}

The starting point in our examination of the theory of rates in the reduced instanton approach is the derivation by Kryvohuz ${ }^{\frac{315}{5}}$ for temperatures above and below $T_{\mathrm{c}}$. A detailed introduction to the underlying theory can be found in these references and will not be repeated here.

In the results section, we use the following formula ${ }^{[5}$ for reaction rate constants at temperatures above $T_{\mathrm{c}}$ (or, equivalently, for $\beta<\beta_{\mathrm{c}}=1 / k_{\mathrm{B}} T_{\mathrm{c}}$ )

$$
k=\frac{1}{Q_{r}} \frac{e^{-\beta V_{0}}}{2 \beta_{\mathrm{c}} \sin \left(\beta / \beta_{\mathrm{c}}\right)} \Delta \sqrt{\frac{\pi}{2}}\left(1+\operatorname{erf}\left(-\frac{\Delta}{\sqrt{2}}\right)\right) e^{\Delta^{2} / 2},
$$

where,

$$
\Delta=\frac{\sqrt{\beta \beta_{\mathrm{c}}}}{1}\left(\frac{\beta_{\mathrm{c}}^{2}}{\beta^{2}}-1\right) \sqrt{-\frac{d E_{b}\left(\beta_{\mathrm{c}}\right)}{d \beta}} .
$$

The symbols of Equation (1) are the reactant state partition function $Q_{\mathrm{r}}$, the reciprocal temperature $\beta=1 / k_{\mathrm{B}} T$, the potential at the barrier top $V_{0}$, and the rate of change of the tunnelling energy $E_{b} \equiv E_{b}(\beta) ; 0<E_{b}(\beta)<V_{0}: \forall \beta>\beta_{\mathrm{c}}$. We assume that the reactant state energy is 0 .

Our implementation of this established theory differs in the computation of certain components, specifically, the rate of change of the tunnelling energy $E_{b}$ as a function of the inverse 
temperature $\beta$ and in the technical aspects of calculating the contribution to the partition function due to the action from tunnelling orthogonal to the instanton path. We explore how to calculate the action from orthogonal oscillations in section 2.1 before examining a simple technique to approximate $d E_{b} / d \beta$.

In the reduced instanton theory, the rate constant at temperatures below $T_{\mathrm{c}}$ is given by ${ }^{3}$

$$
k=\frac{1}{2 Q_{\mathrm{r}}} \sqrt{\frac{-1}{2 \pi}\left(\frac{d E_{b}}{d \beta}+\frac{d^{2} \sigma}{d \beta^{2}}\right)} e^{-S_{\mathrm{E}}-\sigma} \times\left(1+\operatorname{erf}\left(\frac{\left(V_{0}-E_{b}\right)}{\sqrt{2}}\left(-\frac{d E_{b}}{d \beta}-\frac{d^{2} \sigma}{d \beta^{2}}\right)^{-1 / 2}\right)\right)
$$

We do not include zero-point corrections to $V_{0}$ or $E_{b}$ in Equation (2) as these corrections cancel near $T_{c}$ or become irrelevant at low temperatures due to the term $d E_{b} / d \beta+d^{2} \sigma / d \beta^{2}$ rapidly approaching zero. New terms appearing in Equation (2) that are absent from canonical instanton theory are the classical action $S_{\mathrm{E}}$ and the contribution to the instanton partition function by fluctuations orthogonal to the instanton trajectory $\sigma$.

It is useful to define the euclidean action $S_{\mathrm{E}}$ as an integral in imaginary time along an arbitrary path $\mathbf{y}$ in the inverted, multidimensional potential $V(\mathbf{y}(\tau))$. In mass-weighted coordinates it is given by

$$
S_{\mathrm{E}}[\mathbf{y}]=\int_{0}^{\beta}\left(\frac{1}{2}\left(\frac{d \mathbf{y}}{d \tau}\right)^{2}+V(\mathbf{y}(\tau))\right) d \tau
$$

The instanton path is a closed path that satisfies $\delta S_{\mathrm{E}}[\mathbf{y}]=0$. Its length is non-zero for all $\beta>\beta_{\mathrm{c}}$. In our implementation, the vector corresponding to the unstable mode at the transition state is used as a starting guess for the instanton path at some $T \lesssim T_{\mathrm{c}}$. This trajectory is then optimised to meet the condition $\delta S_{\mathrm{E}}[\mathbf{y}]=0$, we denote the coordinates of a point on this optimised path at imaginary time $\tau$ by $\mathbf{y}_{\|}(\tau)$. At lower temperatures, the optimised path at the previous higher temperature is used as the new guess path and is re-optimised. $.22[35$

Unique to the reduced instanton approach is the formulation of $S_{\mathrm{E}}$ as well as explicitly requiring $d E_{b} / d \beta$. We first treat the formulation of $S_{\mathrm{E}}$ and then elaborate on an approximation for $d E_{b} / d \beta$. 


\subsection{Action formulation}

Once the optimisation condition has been reached, the instanton action $S$ can be expanded into a classical component parallel to the instanton trajectory and a component accounting for fluctuations orthogonal to the instanton trajectory. $33 \mid 36$

$$
\begin{aligned}
S & =S_{\mathrm{E}}+S_{\perp} \\
S_{\mathrm{E}} & =\int_{0}^{\beta}\left(\frac{1}{2}\left(\frac{d}{d \tau} \delta \mathbf{y}_{\|}\right)^{2}+V\left(\mathbf{y}_{\|}(\tau)\right)\right) d \tau \\
S_{\perp} & =\int_{0}^{\beta} \frac{1}{2}\left(\frac{d}{d \tau} \delta \mathbf{Y}_{\perp}\right)^{2} d \tau+\int_{0}^{\beta} \frac{1}{2} \delta \mathbf{Y}_{\perp}(\tau)^{T} \cdot \tilde{\mathbf{V}}^{\prime \prime}\left(\mathbf{y}_{\|}(\tau)\right) \cdot \delta \mathbf{Y}_{\perp}(\tau) d \tau
\end{aligned}
$$

In Equation (4) and henceforth, a co-moving coordinate system is used: $\mathbf{y}_{\|}(\tau) \in \mathbb{R}^{N \times 1}$ and $\mathbf{Y}_{\perp}(\tau) \in \mathbb{R}^{N \times(N-1)}$, where $N$ is the dimension of the system. $\mathbf{Y}_{\perp}(\tau)$ is a matrix of the other $N-1$ vectors orthogonal to $\mathbf{y}_{\|}(\tau+d \tau)-\mathbf{y}_{\|}(\tau)$. The fluctuations $\delta \mathbf{y}_{॥}(\tau)$ and $\delta \mathbf{Y}_{\perp}(\tau)$ are respectively, the scalar displacement along the path and an $N-1$ vector of displacements orthogonal to the path. $\tilde{\mathbf{V}}^{\prime \prime}\left(\mathbf{y}_{\|}(\tau)\right) \in \mathbb{R}^{(N-1) \times(N-1)}$ is a reduced, rotated hessian, obtained by projecting $\mathbf{V}^{\prime \prime}\left(\mathbf{y}_{\| 1}(\tau)\right)$ onto the $\mathbf{Y}_{\perp}(\tau)$ basis.

The instanton path is discretised into $P$ images. The integrals in Equation (4) are thus transformed into sums, therefore $d \tau \rightarrow \Delta \tau=\beta / P$. This has the further consequence that as temperature decreases, images accumulate near the ends of the instanton path, leaving the region around the transition state less well modelled.

The integral $S_{\mathrm{E}}$ of Equation (4) is discretised into a Riemann sum:

$$
S_{\mathrm{E}}=\beta \sum_{j=1}^{P}\left(\frac{P}{2 \beta^{2}}\left|\mathbf{y}_{\Perp, j+1}-\mathbf{y}_{\|, j}\right|^{2}+\frac{V\left(\mathbf{y}_{\Perp, j}\right)}{P}\right),
$$

moreover, $e^{\sigma}$ is the contribution to the partition function from fluctuations orthogonal to the instanton path

$$
e^{-\sigma}=\prod_{i=1}^{N-1} \int d \delta \mathbf{Y}_{\perp, \mathbf{0}}{ }^{(i)} \int_{\mathbf{Y}_{\perp}(0)=\mathbf{Y}_{\perp, \mathbf{0}}}^{\mathbf{Y}_{\perp}(\beta)=\mathbf{Y}_{\perp, \mathbf{0}}} \mathcal{D}\left[\delta \mathbf{Y}_{\perp}(\tau)\right] e^{-S_{\perp}}
$$

where the superscript $(i)$ indicates the $i$ - th vector of the stationary basis $\mathbf{Y}_{\perp, \mathbf{0}}$. Depending upon which temperature regime we are in, either $T \lesssim T_{\text {c }}$ or $T \ll T_{\text {c }}$, we make use of, 
respectively, the stability matrix differential equation or frequency averaging to determine $\sigma$. We also present in the results a third method for calculating $\sigma$ known as eigenvalue tracing, an explanation of this method is given elsewhere ${ }^{32}$ and we present it here for the purpose of comparison.

\subsubsection{The stability matrix differential equation}

A solution for $\sigma$ is found by solving the stability matrix differential equation $33[37$

$$
\begin{aligned}
\frac{d}{d \tau} \mathbf{R}(\tau)+\mathbf{F}(\tau) \cdot \mathbf{R}(\tau) & =0 ; \mathbf{R}(0)=\mathbf{1} \\
\mathbf{R}(\tau) & =\left(\begin{array}{cc}
\mathbf{A}_{1}(\tau) & \mathbf{A}_{2}(\tau) \\
\dot{\mathbf{A}}_{1}(\tau) & \dot{\mathbf{A}}_{2}(\tau)
\end{array}\right),
\end{aligned}
$$

where

$$
\mathbf{F}(\tau)=\left(\begin{array}{cc}
\mathbf{0} & \mathbf{- 1} \\
\mathbf{V}^{\prime \prime}(\tau) & \mathbf{0}
\end{array}\right)
$$

The stability matrix differential Equation (7), when solving for $\mathbf{A}_{1}$ or $\mathbf{A}_{2}$, is known as the the Gel'fand-Yaglom equation.

The stability parameters (eigenvalues) $u_{i}$ of a matrix $\mathbf{M}$, where $\mathbf{R}(\beta)=e^{\mathbf{M}}$, are sought. These uniquely determine $\sigma$

$$
\sigma=\sum_{i=1}^{\mathfrak{N}} \ln \left(2 \sinh \frac{u_{i}}{2}\right) ; \mathfrak{N}=\operatorname{dim}(\mathbf{M}) / 2
$$

The dimension of $\mathbf{F}, \mathbf{R}$ and $\mathbf{M}$ is either $2 N$ or $2(N-1)$. In the former case, two extra zeros appear in the eigenvalue spectrum of $\mathbf{M}$, these eigenvalues are excluded from the sum in Equation (9). In the latter, we must use a reduced, rotated potential Hessian $\tilde{\mathbf{V}}^{\prime \prime}(\tau)$. It is constructed as follows.

At each image we solve the eigenvalue equation $\mathbf{V}^{\prime \prime}(\tau) \cdot \mathbf{X}(\tau)=\boldsymbol{\omega}^{2}(\tau) \mathbf{X}(\tau)$ to find the eigenvectors $\mathbf{X}(\tau)$ of the full potential Hessian. The diagonal matrix $\boldsymbol{\omega}^{2}(\tau)$ contains the force constants. These eigenvectors are projected on to the basis $\mathbf{Y}_{\perp}$, producing the projector matrix $\mathbf{P}(\tau)=\mathbf{X}(\tau) \cdot \mathbf{Y}_{\perp}(\tau)$. We then determine $\tilde{\mathbf{V}}^{\prime \prime}$ according to:

$$
\tilde{\mathbf{V}}_{N-1 \times N-1}^{\prime \prime}(\tau)=\mathbf{P}_{N-1 \times N}^{T}(\tau) \cdot \boldsymbol{\omega}(\tau)^{2} \cdot \mathbf{P}_{N \times N-1}(\tau)
$$


To begin, we choose an arbitrary starting image on the instanton path which is assumed to correspond to $\tau=0$. At this image, an initial guess basis is supplied to a Gram-Schmidt algorithm. For this, all but one of the eigenvectors $\mathbf{X}(0)$ and the normalised vector $\mathbf{y}_{\Perp}(\Delta \tau)-$ $\mathbf{y}_{\Perp}(0)$ are used as the initial guess of the basis for $\mathbf{Y}_{\perp}(0)$. The excluded eigenvector is the one that has the largest projection onto $\mathbf{y}_{\|}(\Delta \tau)-\mathbf{y}_{\|}(0)$. Our implementation of the GramSchmidt algorithm ensures that the normalised vector $\mathbf{y}_{\Perp}(\tau+\Delta \tau)-\mathbf{y}_{\|}(\tau)$ is always a part of the orthogonal co-moving basis at every image. For subsequent co-moving bases $\mathbf{Y}_{\perp}(\tau)$, the new guess basis again consists of the corresponding $\mathbf{y}_{\|}(\tau+\Delta \tau)-\mathbf{y}_{\|}(\tau)$, yet the guess for the remaining $N-1$ basis vectors are the eigenvectors of the Hessian at the previous image. In this way, our co-moving basis retains a maximal degree of coherence which makes possible the comparison of the eigenvectors of $\tilde{\mathbf{V}}^{\prime \prime}$ of neighbouring images and permits the tracing of the eigenvalues of $\tilde{\mathbf{V}}^{\prime \prime}$ along the instanton path.

The ansatz for eigenvalue tracing lies in the assumption that the stability parameters $u_{i}$ of Equation (9) can be interpreted as frequencies orthogonal to, and averaged along, the instanton path. The coherent nature of the proposed co-moving basis means that each eigenvector of one particular Hessian is almost parallel to one (and only one) eigenvector of a neighbouring Hessian. We can then ascribe the eigenvalues of these corresponding eigenvectors to the same orthogonal mode, the average frequency of this mode along the path gives the required stability parameter. 32

Directly solving the stability matrix is a reliable technique in a temperature range near, but below the crossover temperature, where instanton paths are short. The range of applicability of the stability matrix as a method for obtaining $\sigma$ will be analysed in the discussion.

\subsubsection{Frequency averaging}

Below a certain system-dependent temperature, the stability matrix method is unable to reliably calculate $\sigma$. In the low temperature limit the eigenvalues $u_{i}$, of Equation (9), exhibit an increasingly linear dependence on $\beta$. We thus rewrite $u_{i}=\beta \omega_{\perp, i}$, hence Equation (9) becomes

$$
\lim _{\beta \rightarrow \infty} \sigma \sim \sum_{i=1}^{\mathfrak{N}} \frac{\beta \bar{\omega}_{\perp, i}}{2}
$$


where $\mathfrak{N}$ is set to $N-1$. The set of frequencies $\bar{\omega}_{\perp}$ are average frequencies of the reduced, rotated Hessians $\tilde{\mathbf{V}}^{\prime \prime}$. By analyzing Equation (4), the following derivations justify its usage in the low temperature regime. The kinetic part of Equation (4) can be partially integrated

$$
\int_{0}^{\beta} \frac{1}{2}\left(\frac{d}{d \tau} \delta \mathbf{Y}_{\perp}\right)^{2} d \tau=\left.\frac{1}{2} \frac{\delta \mathbf{Y}_{\perp}^{T}}{d \tau} \cdot \delta \mathbf{Y}_{\perp}(\tau)\right|_{0} ^{\beta}-\int_{0}^{\beta} \frac{1}{2} \delta \mathbf{Y}_{\perp}(\tau)^{T} \cdot \frac{d^{2}}{d \tau^{2}} \delta \mathbf{Y}_{\perp} d \tau
$$

The first term on the right is zero due to the boundary conditions $\delta \mathbf{Y}_{\perp}(0)=\delta \mathbf{Y}_{\perp}(\beta)$, this is the condition of a closed instanton path. Equation (4) can be rewritten

$$
S_{\perp}=\int_{0}^{\beta} \delta \mathbf{Y}_{\perp}(\tau)^{T} \cdot\left(-\frac{1}{2} \frac{d^{2}}{d \tau^{2}}+\frac{1}{2} \tilde{\mathbf{V}}^{\prime \prime}\left(\mathbf{y}_{\|}(\tau)\right)\right) \cdot \delta \mathbf{Y}_{\perp}(\tau) d \tau
$$

The term in brackets in Equation (13) is the differential equation for uncoupled, quantum harmonic oscillators. This can be replaced with its diagonal eigenvalue matrix:

$$
S_{\perp}=\int_{0}^{\beta} \delta \mathbf{Y}_{\perp}(\tau)^{T} \cdot \frac{\tilde{\Omega}(\tau)}{2} \cdot \delta \mathbf{Y}_{\perp}(\tau) d \tau
$$

In the limit $\beta \rightarrow \infty$, only the ground states of the uncoupled quantum harmonic oscillators make a significant contribution to the partition function, thus the elements of $\Omega(\tau)$ are $\Omega(\tau)_{n m}=\delta_{n m} \omega_{n m}(\tau)$. Discretising the integral in Equation (14) one can rewrite Equation (6)

$$
e^{\sigma}=\prod_{i=1}^{\mathfrak{N}} \int d \delta \mathbf{Y}_{\perp, \mathbf{0}}{ }^{(i)}\left[\delta \mathbf{Y}_{\perp, \mathbf{0}}{ }^{(i) T} \cdot e^{\sum_{j=1}^{P}\left(\frac{\tilde{\Omega}^{(j)}}{2}\right) \Delta \tau} \cdot \delta \mathbf{Y}_{\perp, \mathbf{0}}{ }^{(i)}\right]
$$

We are left with the definition of an operator trace. This formulation for $\sigma$ has been used elsewhere. [

$$
\begin{aligned}
e^{\sigma} & =\int d \delta \mathbf{Y}_{\perp, \mathbf{0}}\left\langle\delta \mathbf{Y}_{\perp, \mathbf{0}}\right| e^{\sum_{j=1}^{P} \Delta \tau \frac{\tilde{\mathbf{\Omega}}^{(j)}}{2}\left|\delta \mathbf{Y}_{\perp, \mathbf{0}}\right\rangle}, \\
\sigma & \sim \frac{\beta}{2 P} \sum_{j=1}^{P / 2} \operatorname{tr}\left(\tilde{\boldsymbol{\Omega}}^{(j)}\right) .
\end{aligned}
$$

We see that Equation (16) corresponds exactly to Equation (11) given the trace is conducted over $\mathfrak{N}$ dimensions. As with the stability matrix method, either full-dimensional Hessians or the reduced, rotated Hessians can be used to find $\sigma$. Using the full-dimensional Hessians means $\mathfrak{N}=N$, in which case one adds a correction to Equation (16):

$$
\sigma \sim \frac{\beta}{2 P} \Re\left[\sum_{j=1}^{P / 2} \operatorname{tr}\left(\boldsymbol{\Omega}^{(j)}\right)-\sqrt{\left\langle\mathbf{y}_{\Perp, j}-\mathbf{y}_{\Perp, j+1}\left|\mathbf{V}^{\prime \prime}{ }_{j}\right| \mathbf{y}_{\Perp, j}-\mathbf{y}_{\Perp, j+1}\right\rangle}\right],
$$


This removes any contribution to $\sigma$ from oscillations parallel to the instanton path. Equation (16) (or Equation (17) ) is a convenient form for $\sigma$, it is simply a sum of all the eigenvalues from the Hessians at each image. It should also be noted that for computational purposes, one should take only the real part of the RHS of Equation (17) because, for a small $P$, spurious, complex contributions to $\sigma$ tend to increase.

As opposed to unimolecular reactions, calculation of both reactant and instanton partition functions in bimolecular reactions demand a different approach to the treatment of very small frequencies at low temperatures. In unimolecular reactions, since the images of the instanton path accumulate near the reactant state, the conditions imposed on the treatment of small frequencies in the reactant state partition function can be equally applied to small frequencies in the instanton partition function. In bimolecular reactions, at low temperatures, the images of the instanton path may accumulate near a pre-reactive minimum, the coordinate and potential landscape of which is likely very different when compared with the reactant state of the separated system. The conditions for eliminating small frequencies from the instanton partition thus need modifying. In the calculations shown for the reaction $\mathrm{H}_{2}$ $+\mathrm{OH} \rightarrow \mathrm{H}+\mathrm{H}_{2} \mathrm{O}$ in the frequency averaging scheme, we omit from Equation (16) and Equation (17) those frequencies where $\beta \bar{\omega}_{\perp, i} / 2<\sinh ^{-1}(1 / 2)$. This is justified since

$$
\left.\begin{array}{rlc}
\operatorname{sgn}(\log [2 \sinh (x)]) & =\operatorname{sgn}(x) \\
\log [2 \sinh (x)] & \sim & x
\end{array}\right\} \forall: x>\sinh ^{-1}(1 / 2) .
$$

The second condition of Equation $(18)$ is in any case the main assumption behind the frequency averaging approach. It is natural to ask at which $\beta$ the stability matrix approach should give way to frequency averaging. The reliability of the stability matrix approach depends strongly on the length of the path, which, depending on the potential, can change drastically between two similar temperatures. From Equation (18) we know that frequency averaging is maximally inaccurate when $\beta=\beta_{c}$, we can ask the more instructive question "what is the upper bound on the relative uncertainty $\delta$ in $\sigma$ when calculated using frequency averaging?" This upper bound is given by

$$
\delta\left(\beta>\beta_{c}\right)<\frac{\sum_{i=1}^{\mathfrak{N}} \log \left(1-e^{-\beta_{c} \bar{\omega}_{\perp, i}^{\mathrm{TS}}}\right)}{\sum_{i=1}^{\mathfrak{N}} \beta_{c} \bar{\omega}_{\perp, i}^{\mathrm{TS}} / 2} .
$$


As an example, for the reaction methylhydroxycarbene $\rightarrow$ acetaldehyde treated in the results we get $\delta\left(\beta_{c}\right)=4.97 \%$.

Furthermore, in the version implemented in DL-FIND, the zero eigenvalues of $\mathbf{M}(\beta)$ (identified by projection on to the path) are displayed in the output. The user may, independently of Equation (19), decide at which temperature these zero eigenvalues are no longer negligible and hence determine the temperature at which the calculation of $\sigma$ using the eigenvalues of the stability matrix are no longer reliable.

Having defined how the action enters into Equation (2) we now explore the methods available for deriving $d E_{b} / d \beta$.

\subsection{Temperature dependence of the tunnelling energy}

The most accurate approach to determine $d E_{b} / d \beta$ is a finite difference procedure. To this end, the instanton path is first optimised at two nearby temperatures. The endpoints of the path lie at a particular energy on the potential surface, this energy is the tunnelling energy $E_{b}$. A simple ratio between $\Delta E_{b}$ and $\Delta \beta$ for the two paths is used to approximate $d E_{b} / d \beta$.

Re-optimising the instanton path for small temperature changes around the desired temperature can be time consuming. Instead we provide an approximate formula for estimating $d E_{b} / d \beta$ which is sufficiently accurate for all temperatures $T<T_{\mathrm{c}}$.

From the stationary condition for locating instantons, $\delta S_{\mathrm{E}}=0$, one can derive the following relation $22[33$

$$
\beta=\int_{s_{a}\left(E_{b}\right)}^{s_{b}\left(E_{b}\right)} \sqrt{\frac{2}{V(\mathbf{y}(s))-E_{b}}}\left|\frac{d \mathbf{y}(s)}{d s}\right| d s,
$$

where $V\left(\mathbf{y}\left(s_{a}\right)\right)=V\left(\mathbf{y}\left(s_{b}\right)\right)=E_{b}$. For all $s \in \mathbb{R} \mid s_{a}<s<s_{b}$, the tunnelling energy is less than the potential energy. We approximate the integral at this point by expanding the potential in a Taylor series. The expansion is fixed around the reactant and product state coordinates, ensuring the proper behaviour of $d E_{b} / d \beta$ at both limits $\beta \rightarrow \infty$ or $\beta \rightarrow \beta_{\mathrm{c}}$. Moreover, Equation (20) remains unchanged if the radicand is an absolute value

$$
\begin{aligned}
\beta & \sim \int_{s_{a}\left(E_{b}\right)}^{s_{b}\left(E_{b}\right)} \sqrt{\frac{2}{\left|\left(V_{\mathrm{RS}}-E_{b}\right)+\mathbf{y}(s)^{T} \cdot \frac{\mathbf{V}^{\prime \prime} \mathrm{RS}}{2} \cdot \mathbf{y}(s)\right|} \mid}\left|\frac{d \mathbf{y}(s)}{d s}\right| d s . \\
& +\int_{s_{a}\left(E_{b}\right)}^{s_{b}\left(E_{b}\right)} \sqrt{\frac{2}{\left|\left(V_{\mathrm{PS}}-E_{b}\right)+\mathbf{y}(s)^{T} \cdot \frac{\mathbf{V}^{\prime \prime} \mathrm{PS}}{2} \cdot \mathbf{y}(s)\right|} \mid} \frac{d \mathbf{y}(s)}{d s} \mid d s .
\end{aligned}
$$


If we are dealing with asymmetric potentials, the second part of Equation (21) will always be much smaller than the first part. Because the limits of the integral themselves depend on the tunnelling energy one would ordinarily use the Leibniz integral rule to find the derivative with respect to $E_{b}$. However, we retain only the following term in the Leibniz integral rule

$$
\frac{d \beta}{d E_{b}} \sim-\sqrt{\frac{2}{\left|\left(V_{\mathrm{RS}}-E_{b}\right)+\mathbf{y}\left(s_{a}\right)^{T} \cdot \frac{\mathbf{V}^{\prime \prime}{ }_{\mathrm{RS}}}{2} \cdot \mathbf{y}\left(s_{a}\right)\right|}} \times \frac{d\left|\mathbf{y}\left(s_{a}\right)\right|}{d E_{b}},
$$

we may thereby provide a good approximation to full Leibniz rule derivation of $d \beta / d E_{b}$ for most tunnelling energies and remain closer the finite difference estimate for tunnelling energies approaching $V_{\mathrm{RS}}$. The vector $\mathbf{y}\left(s_{a}\right)$ connects the expansion coordinate to that image on the instanton path nearest the reactant state. Furthermore, we may freely choose the parameterisation, and set $s_{a}=\left|\mathbf{y}\left(s_{a}\right)\right|$ and $\left|\frac{d \mathbf{y}\left(s_{a}\right)}{d s}\right|=1$ for all tunneling energies. We make use again of the Taylor series approximation to find a closed form for $d\left|\mathbf{y}\left(s_{a}\right)\right| / d E_{b}$.

$$
\begin{aligned}
& E_{b}-V_{\mathrm{RS}} \sim \mathbf{y}\left(s_{a}\right)^{T} \cdot \frac{\mathbf{V}^{\prime \prime} \mathrm{RS}}{2} \cdot \mathbf{y}\left(s_{a}\right) \\
& E_{b}-V_{\mathrm{RS}} \sim\left|\mathbf{y}\left(s_{a}\right)\right|^{2}\left(\hat{\mathbf{y}}\left(s_{a}\right)^{T} \cdot \frac{\mathbf{V}^{\prime \prime}{ }_{\mathrm{RS}}}{2} \cdot \hat{\mathbf{y}}\left(s_{a}\right)\right), \\
& \frac{d E_{b}}{d\left|\mathbf{y}\left(s_{a}\right)\right|} \sim\left|\mathbf{y}\left(s_{a}\right)\right|\left(\hat{\mathbf{y}}\left(s_{a}\right)^{T} \cdot \mathbf{V}^{\prime \prime}{ }_{\mathrm{RS}} \cdot \hat{\mathbf{y}}\left(s_{a}\right)\right) .
\end{aligned}
$$

Combining Equation (22) and Equation (23) provides reasonably accurate and stable results for all temperatures below $T_{\mathrm{c}}$. We note that this formulation is reasonable for asymmetric barriers because the leading order contribution to the integral in Equations (20) and (21) comes from a small region near the reactant state, or equivalently, when $s \sim s_{a}$. For nearly symmetric barriers, one should multiply Equations (21) and (22) by a factor of 2 .

As the temperature approaches the crossover temperature, a more accurate method ${ }^{5}$ is employed to determine $d E_{b} / d \beta$. Since all images of the instanton collapse to a point when $T=T_{\mathrm{c}}$, Equation (3) simplifies greatly, i.e. the kinetic part is zero. Using the fact that $d^{2} S_{\mathrm{E}} / d \beta^{2}=d E_{b} / d \beta$ and expanding the potential in higher orders, ${ }^{\sqrt[5]{17}}$ one can modify Equation (22) for very short tunnelling paths in order to continue calculating rate constants near $T_{\mathrm{c}}$. To accomplish this and to guarantee smoothness of $d E_{b} / d \beta$ over all temperatures below $T_{\mathrm{c}}$, we use a weighted combination of $d E_{b} / d \beta$ calculated by expanding the potential around the reactant state i.e. Equation 22 and the method expounded in 


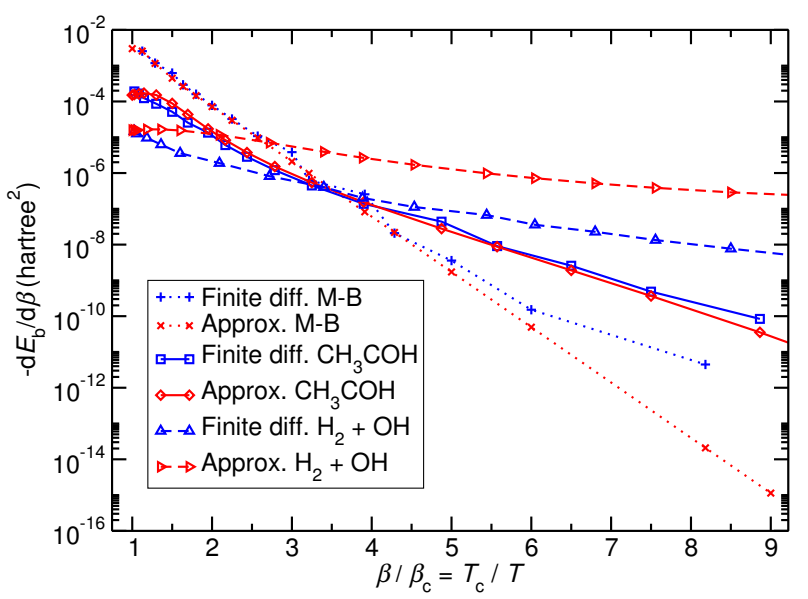

Figure 1: Temperature dependence of the tunnelling energy in the reaction of each of the reactions examined in section 3, red lines were calculated using Equation (22).

Cao and Voth ${ }^{17}$ and Kryvohuz ${ }^{5}$ wherein paths are represented as a fourier series, permitting a closed expression for $d E_{b} / d \beta$ depending on anharmonic terms in the taylor series for the potential around the transition state. With respect to the distance between the reactant state coordinate and the coordinate of that image on the instanton path nearest the reactant state, the weighting of the method in Cao and Voth ${ }^{17}$ and Kryvohuz ${ }^{5}$ increases with the inverse cube of this distance whereas the weighting of Equation (22) drops linearly with this distance. A comparison is shown in Fig. 1 between the finite difference and approximation approaches for the reactions examined in the results.

Clearly the approximation method for $d E_{b} / d \beta$ in the reaction $\mathrm{H}_{2}+\mathrm{OH} \rightarrow \mathrm{H}+\mathrm{H}_{2} \mathrm{O}$ does not agree as nicely with the finite-difference method as do the other two cases. The potential landscape around the reactant state for the reaction on the Müller-Brown surface and for methylhydroxycarbene, are quite deep and well approximated by a harmonic potential $\left(2^{\text {nd }}\right.$ order Taylor series) in comparison to the reaction $\mathrm{H}_{2}+\mathrm{OH} \rightarrow \mathrm{H}+\mathrm{H}_{2} \mathrm{O}$. The approximations used in Equation (21) and Equation (23) are made only to second order. This suggests that $d E_{b} / d \beta$, when calculated by the given approximation, and applied to reactions with shallow reactant states or pre-reactive minima will not be well modelled except at temperatures close to $T_{c}$. Fortunately, the rate constant is not overly sensitive to inaccuracies in $d E_{b} / d \beta$ since it enters the rate equation under the square root sign. 


\subsection{A correction to canonical instanton theory close to $T_{\mathrm{c}}$}

As mentioned in the introduction, an endemic problem of canonical instanton theory is the overestimation of rate constants at temperatures approaching $T_{\mathrm{c}}$. The derivation of rate Equation (2) corrects this behaviour by ensuring those tunnelling paths with energies $E_{b}>V_{0}$ do not contribute to the rate calculation.

A better understanding of this peculiarity can be gained by examining the equation for the canonical instanton theory rate $\left(k_{\text {inst }}\right)$ and the expression for the flux $(f)$ over a barrier used to determine the rate in Equation (2)

$$
\begin{aligned}
k_{\text {inst }} & =\frac{2}{\beta} \mathfrak{I}(\log (Q)) \sim \frac{2}{\beta} \frac{\mathfrak{I}\left(Q_{\text {inst }}\right)}{Q_{r}}, \\
k & =\frac{f}{Q_{r}}=\frac{1}{2 \pi Q_{r}} \int_{-\infty}^{V_{0}} e^{-\beta E} \sum_{k=1}^{\infty}(-1)^{k-1} e^{-k S_{0}(E)} \prod_{i=1}^{N} \frac{1}{2 \sinh k u_{i} / 2} d E,
\end{aligned}
$$

where $Q_{\text {inst }}$ is the partition function of the instanton, $S_{0}$ is the shortened action and the term under the sum is the cumulative reaction probability. ${ }^{[}$At low temperatures it is sufficient to truncate the sum over $k$ to only the first term, furthermore, the term under the product should look familiar from Equation (9), thus we may simplify.

$$
k=\frac{f}{Q_{r}}=\frac{1}{2 \pi Q_{r}} e^{-\sigma} \int_{-\infty}^{V_{0}} e^{-\beta E} e^{-S_{0}(E)} d E,
$$

As explained in the appendix of Ref. 3, $S_{0}$ in Equation (24) can be expanded to second order around $E=E_{b}$, which results in an expression containing the error function, indeed this is the term in the large braces in Equation (2).

In canonical instanton theory, the partition function of the instanton $\left(Q_{\text {inst }}\right)$ is expressed as an integral in configuration space over all possible closed paths. At temperatures approaching $T_{\mathrm{c}}$ an increasing number of closed paths possess an energy $E_{b}>V_{0}$. There is no way to selectively remove these paths from the integral in canonical instanton theory. This problem persists for all $T<T_{\mathrm{c}}$, however, the contribution to the partition function from paths with $E_{b}>V_{0}$ as $T \rightarrow 0 \mathrm{~K}$ becomes vanishingly small. When using the expression for the flux, we can explicitly control which states contribute to the rate expression by setting the upper limit of the integral to $V_{0}$, this effectively truncates the Boltzmann distribution for the state occupancy to zero for those states with energy greater than the barrier height. This is a reasonable step to take, since those states face no restriction to recrossing. 
If however we replace the upper limit of the integral in Equation $(24)$ with $\infty$ the error function becomes 1, with the rate expression simplifying to

$$
k=\frac{1}{Q_{\mathrm{r}}} \sqrt{\frac{-1}{2 \pi}\left(\frac{d E_{b}}{d \beta}+\frac{d^{2} \sigma}{d \beta^{2}}\right)} e^{-S_{\mathrm{E}}-\sigma} .
$$

In this case, as was shown in a paper by Althorpe ${ }^{21}$, the canonical instanton rate $k_{\text {inst }}$, and the rate $k$ determined by Equation (26) are equivalent and both would exhibit the same overestimation of the rate near $T_{c}$.

The error function in Equation (2) takes on the role of a correction factor and may be used to modify the results of a rate calculation using canonical instanton theory $\left(k_{\text {inst }}\right)$ in exactly the same way it modifies the rate calculated using the flux over the barrier approach

$$
k_{\text {corr }}=k_{\text {inst }} \frac{1}{2}\left(1+\operatorname{erf}\left(\frac{\left(V_{0}-E_{b}\right)}{\sqrt{2}}\left(-\frac{d E_{b}}{d \beta}\right)^{-1 / 2}\right)\right) .
$$

At $T=T_{\text {c }}$ we have $E_{b}=V_{0}$ so that $k_{\text {corr }}=k_{\text {inst }} / 2$. At low temperature, $E_{b} \ll V_{0}$ and the correction factor is almost unity, $k_{\text {corr }} \approx k_{\text {inst }}$.

\subsection{Rate constant calculations with Bell and Eckart approximations}

We compare our instanton rate constants to one-dimensional tunneling approximations and to rate constants obtained without tunneling. Even though these approaches have been used in our group's previous work, $29 \sqrt{39}, \underline{45}$ they have not yet been described in detail. In harmonic transition state theory, the potential energy surface around the reactant and the transition structure is approximated harmonically. The vibrational frequencies of the reactant are denoted $\omega_{\mathrm{RS}, i}$ and those of the transition state $\omega_{\mathrm{TS}, i}$. For a system with $D$ vibrational degrees of freedom, the rate constant in harmonic transition state theory is

$$
k_{\mathrm{HTST}}(\beta)=\frac{1}{2 \pi \beta \hbar} \frac{Q_{\mathrm{rot}, \mathrm{TS}}}{Q_{\mathrm{rot}, \mathrm{RS}}} \frac{Q_{\mathrm{trans}, \mathrm{TS}}}{Q_{\mathrm{trans}, \mathrm{RS}}} \frac{\prod_{i=1}^{D} 2 \sinh \left(\beta \hbar \omega_{\mathrm{RS}, i} / 2\right)}{\prod_{i=1}^{D-1} 2 \sinh \left(\beta \hbar \omega_{\mathrm{TS}, i} / 2\right)} \exp \left(-\beta V_{0}\right) .
$$

The rotational partition function is denoted $Q_{\text {rot }}$ and the translational partition function $Q_{\text {trans }}$. Equation 28 can be used for uni-molecular (in which case the ratio $Q_{\text {trans,Ts }} / Q_{\text {trans,RS }}$ is unity) as well as bimolecular rate constants. All vibrational degrees of freedom are treated as quantum harmonic oscillators. Thus, Equation (28) includes effects of the vibrational 
zero point energy in the harmonic approximation, but neglects tunneling. Is is denoted as "Classical + ZPE" in the following.

In a coarse approximation, tunneling can be incorporated by using a one-dimensional tunneling correction, i.e., by assuming that tunneling happens only along the reaction coordinate and the vibrational levels are not affected. Then $k_{\text {Eckart }}$, the rate constant including tunneling with the Eckart approximation, as used in the present work, is

$$
k_{\text {Eckart }}=k_{\mathrm{HTST}} \kappa_{\text {Eckart }}
$$

where $\kappa_{\text {Eckart }}$ is the tunneling correction factor. It is calculated as fraction of the quantum flux and the classical flux through the barrier:

$$
\kappa=\frac{f_{\text {quantum }}}{f_{\text {classical }}}=\beta \exp \left(\beta V_{\mathrm{VA}}\right) \int_{0}^{\infty} P(E) \exp (-\beta E) d E .
$$

Here, $V_{\mathrm{VA}}$ is the vibrationally adiabatic barrier, i.e. $V_{0}$ plus the difference in zero point energy between reactant and transition state, $E$ given relative to the vibrationally adiabatic energy of the reactant, and $P(E)$ the quantum mechanical transmission coefficient. For a symmetric Eckart-shaped barrier with the same barrier frequency as the full barrier and the same height as the vibrationally adiabatic barrier, $P_{\text {Eckart }}(E)$ is given as $\underline{46}$

$$
P_{\text {Eckart }}(E)=\frac{\cosh (2 a)-1}{\cosh (2 a)-\cosh (d)}
$$

with

$$
\begin{aligned}
a & =\frac{2 \pi \sqrt{V_{\mathrm{VA}} E}}{\hbar \omega_{b}} \\
d & =\frac{2 \pi \sqrt{4 V_{\mathrm{VA}}^{2}-\hbar \omega_{b} / 4}}{\hbar \omega_{b}}
\end{aligned}
$$

This approach is denoted as Eckart approximation in the following. One could also use the asymmetric Eckart barrier instead of Equation (31), but that would require an additional parameter and generally only marginally improves the accuracy.

Alternatively to the symmetric Eckart barrier, one can also approximate tunneling by the transmission probability of a truncated parabolic barrier with the barrier frequency $\omega_{b}$. Bell derived the tunneling correction factor $\kappa_{\text {Bell }}(T)$ as the exact solution of the permeability of 
a truncated parabolic barrier ( $2^{\text {nd }}$ order polynomial):

$$
\kappa_{\text {Bell }}(T)=\frac{\pi u}{\sin (\pi u)}-u \exp (a-b)\left[\frac{1}{1-u}-\frac{\exp (-b)}{2-u}+\frac{\exp (-2 b)}{3-u}-\ldots\right]
$$

with $u=T_{\mathrm{c}} / T, a=\beta V_{\mathrm{VA}}$, and $b=V_{\mathrm{VA}} /\left(k_{\mathrm{B}} T_{\mathrm{c}}\right)$. Note that $\kappa_{\mathrm{Bell}}(T)$ is finite even for $T \rightarrow T_{\mathrm{c}}$, $T \rightarrow T_{\mathrm{c}} / 2, \ldots$ This approach is denoted "Bell" in the following.

\section{Results}

We now apply the reduced instanton theory and the corrected canonical instanton theory to three different systems: The two-dimensional Müller-Brown potential, the unimolecular reaction methylhydroxycarbene $\rightarrow$ acetaldehyde $(N=21)$ and the bimolecular gas-phase reaction $\mathrm{H}_{2}+\mathrm{OH} \rightarrow \mathrm{H}+\mathrm{H}_{2} \mathrm{O}(N=12)$. The objective is to make a comparison between the reduced instanton theory and canonical instanton theory but also to illustrate how the overestimation of rate constants near $T_{\mathrm{c}}$ is reduced by both this method and by using Equation (27). In the temperature range over $T_{\mathrm{c}}$ we compare with rate constants calculated using classical theories as well as the fitted one-dimensional potentials (Eckart and Bell).

In this work, we compare methods rather than aiming at new insight into specific molecular systems. Therefore, we have chosen systems for which the potential energy and its derivatives can be calculated efficiently and without numerical noise. Instanton theory, however, is applicable efficiently to real-world applications with energies calculated on the fly as we and others have demonstrated frequently in the past.

\subsection{The Müller-Brown surface}

The Müller-Brown surface ${ }^{62}$ is a common "toy model" for testing reaction rate theories. We used the mass of a hydrogen atom and the minimum at the coordinate $(-0.050,0.467)$ as the reactant state. This results in a potential energy barrier of 0.19 Hartree and a crossover temperature of $2207 \mathrm{~K}$. The main advantage of testing on a two dimensional surface comes from the fact that, at each image, there exists only one, two-component vector orthogonal to the instanton path. The utility of this means that there should be no difference between the frequency-averaging approach, or the stability matrix method in the evaluation of $\sigma$ at low 


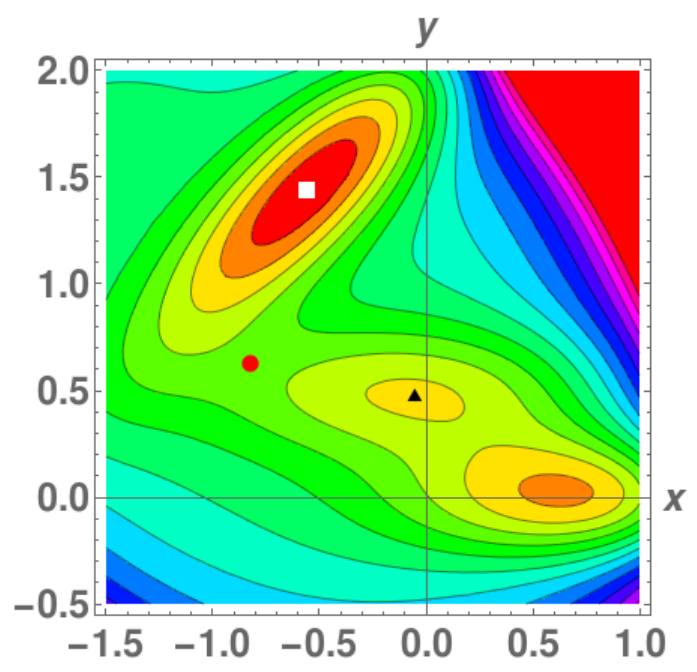

Figure 2: The Müller-Brown PES. The black triangle represents the location of the reactant state $(-0.050,0.467)$, the white square is the global minimum $(-0.558,1.44)$ and the red disc is the transition state $(-0.822,0.624)$. The potential difference between the reactant and transition state is 0.19 Hartree.
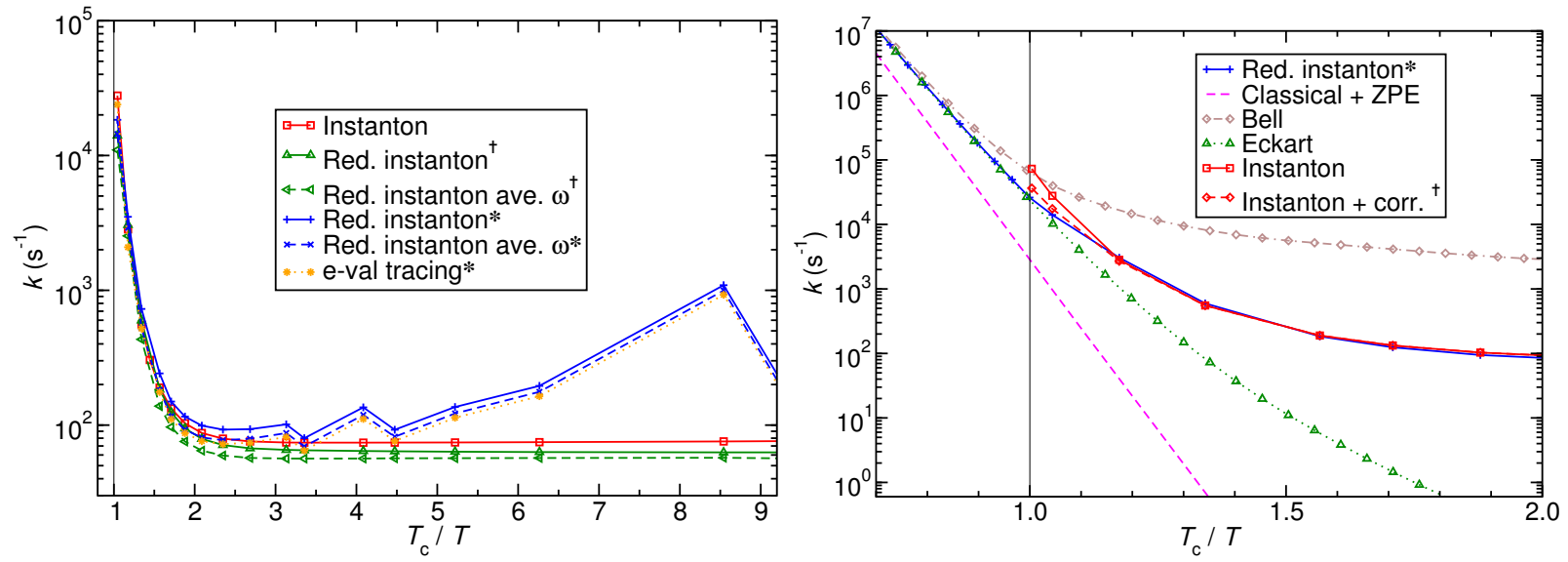

Figure 3: Rate constants for the Müller-Brown potential. Comparison between canonical instanton theory (red line) and reduced instanton theory (blue \& green lines). The symbol * signifies $d E_{b} / d \beta$ was calculated using the finite difference method, $\dagger$ indicates $d E_{b} / d \beta$ was calculated using Equation (22). Red diamonds refer to Equation (27). The top graph shows temperatures below $T_{\mathrm{c}}$, the bottom graph around and above $T_{\mathrm{c}}$. Comparisons to classical transition state theory and the Bell and Eckart approximations are given. 
temperatures, except for small numerical errors arising from the second part of Equation (17) which are caused by the discrete nature of the path.This is indeed the case, as can be seen in Fig. 3 .

Interestingly, due to the fact that the tunnelling energy and the energy of the reactant state are numerically very close, the finite difference method (blue and orange curves, Fig. 3) to determine $d E_{b} / d \beta$ becomes unreliable. This is evidence of the utility of the formula derived in section 2.2 .

For those temperatures near and above $T_{\mathrm{c}}$ (Fig. 3 ) we can see that the reduced instanton theory performs favourably, producing rate constants which smoothly transition towards those found by the formula for rate constants above $T_{\mathrm{c}}$. Also evident is the deviation in the rate constants calculated by canonical instanton theory in this limit, it is clear there is an overestimation of the rate constant near $T_{\mathrm{c}}$ which can be partly corrected by Equation (27).

\subsection{Unimolecular reaction: methylhydroxycarbene $\rightarrow$ acetaldehyde}

The reaction of methylhydroxycarbene to acetaldehyde, $\mathrm{CH}_{3} \mathrm{COH} \rightarrow \mathrm{CH}_{3} \mathrm{CHO}$, via tun-

nelling mechanisms has gained some attention recently. $\underline{6364,23}$ At low temperatures, although a lower barrier towards the formation of vinyl alcohol is present, the formation of acetaldehyde is favoured due to the shorter tunnelling path. This, and the fact that it is a reaction in many degrees of freedom, make it a solid candidate to compare the canonical and reduced instanton theories.

For this work, a local potential energy surface was created by training a neural network to fit the DFT potential used previously ${ }^{23}$ in order to provide a fast and noise-free potential. Possible inaccuracies in the fit should not affect the comparisons performed here. The potential energy barrier height resulted in $133 \mathrm{~kJ} \mathrm{~mol}^{-1}$ and the crossover temperature was $T_{\mathrm{c}}=461 \mathrm{~K}$.

The most obviously striking feature in Fig. 4 of the rate constant obtained from reduced instanton theory for this reaction is the failure of the stability matrix method below about $200 \mathrm{~K}$. At this temperature, the length of the instanton path has stretched to a point that the deviation in the optimised instanton path to the true instanton path is such that a naive solution to Equation (7) would imply that a stable instanton orbit no longer exists. 

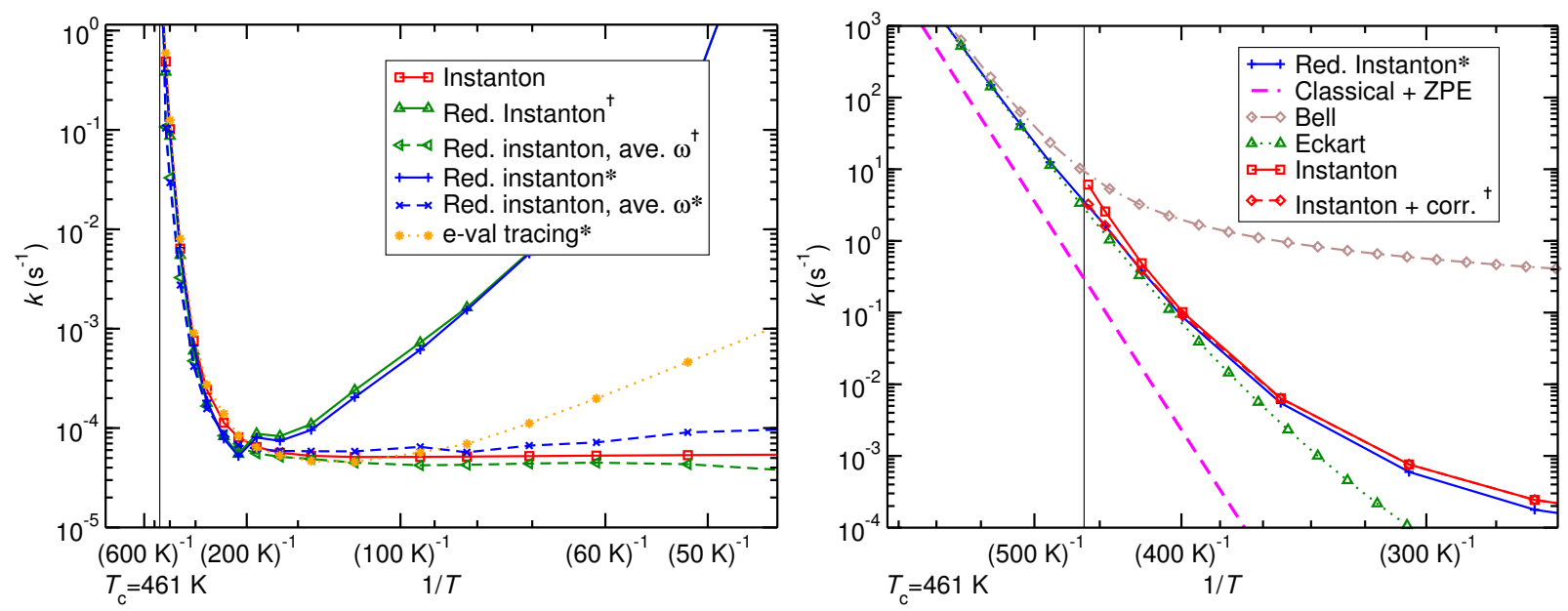

Figure 4: Rate constants for the reaction of methylhydroxycarbene to acetaldehyde below $T_{\mathrm{c}}$ : Comparison between canonical instanton theory (red line) and reduced instanton theory (blue \& green lines). The symbol $*$ signifies $d E_{b} / d \beta$ was calculated using the finite difference method, $\dagger$ indicates $d E_{b} / d \beta$ was calculated using equation Equation (22). Red diamonds refer to Equation (27).

What this means in concrete terms is there are no longer two easily identifiable zero eigenvalues in the spectrum of Equation (9), there remains however two eigenvectors with a considerably larger projection on the instanton path than all other eigenvectors. We identify these eigenvectors and continue to remove their eigenvalues from the sum in Equation (9). This is however only a makeshift solution, as temperatures continue to decrease, all the eigenvectors of the stability matrix exhibit a non-negligible projection onto the instanton path, hence the divergence of the green and blue lines.

Rate constants calculated using the frequency averaging approach for $\sigma$ (left triangles \& crosses) are, as expected, inaccurate when $T \lesssim T_{\mathrm{c}}$. However as temperatures decrease the rate constants become quite stable, and allow the reduced instanton theory to produce valid results even at very low temperatures. Above the crossover temperature in Fig. 4 again a slight reduction in the rate constant is seen in comparison to canonical instanton theory near $T_{\mathrm{c}}$. 

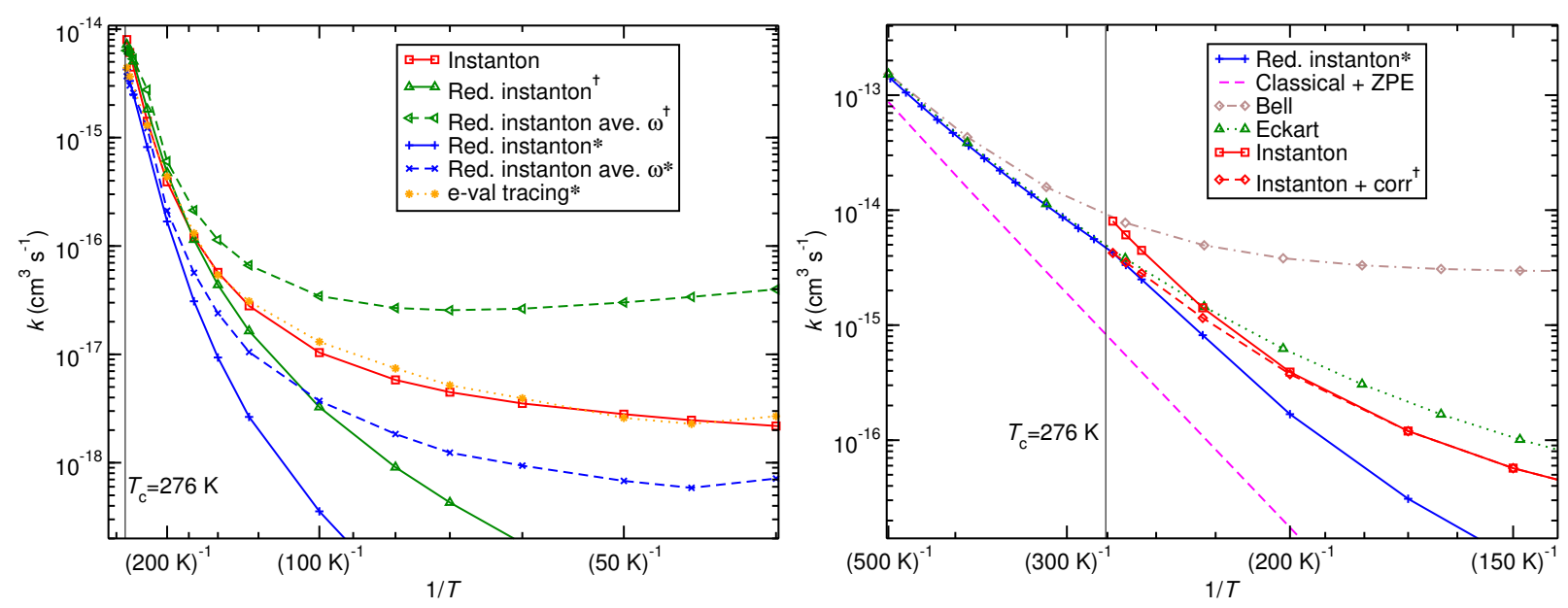

Figure 5: Reaction rate constant for the reaction of $\mathrm{H}_{2}+\mathrm{OH} \rightarrow \mathrm{H}+\mathrm{H}_{2} \mathrm{O}$ below $T_{\mathrm{c}}$ : Comparison between canonical instanton theory (red line) and reduced instanton theory (blue \& green lines). The symbol $*$ signifies $d E_{b} / d \beta$ was calculated using the finite difference method, $\dagger$ indicates $d E_{b} / d \beta$ was calculated using equation Equation (22). Red diamonds refer to Equation (27).

\subsection{Bimolecular reaction: $\mathrm{H}_{2}+\mathrm{OH} \rightarrow \mathbf{H}+\mathbf{H}_{2} \mathrm{O}$}

The final component in our set of systems for method comparison is the bimolecular reaction of $\mathrm{H}_{2}+\mathrm{OH} \rightarrow \mathrm{H}+\mathrm{H}_{2} \mathrm{O}$ in the gas phase. In the interest of providing a rigorous comparison between methods, it is prudent to include a bimolecular type reaction. The rate constants calculated here utilise the NN1 fitted potential energy surface. ${ }^{65}$

This type of reaction is a prototypical reaction for four-atom systems and is also of interest in astrochemistry, where, due to low temperatures, reactions are dominated by tunnelling effects. $\stackrel{43}{4}$

As opposed to the other two test systems, the rate constants obtained using the stability matrix approach tend to deviate from the canonical instanton curve much faster. The rapid departure of the blue curve in Fig. 5 from the red curve is evidence of this. However, since this system already has a quite low crossover temperature $\left(T_{\mathrm{c}}=276 \mathrm{~K}\right)$, we can expect the frequency averaging and eigenvalue tracing approaches to produce reasonable results for all temperatures below $T_{\mathrm{c}}$. As is visible in Fig. 5, usage of eigenvalue tracing produces a smoother transition from below $T_{\mathrm{c}}$ to above $T_{\mathrm{c}}$ than would be possible in reduced instanton 
theory without eigenvalue tracing. At moderate to low temperatures, the rate constants from reduced instanton theory without eigenvalue tracing in Fig. 5 (dashed, blue line) are consistently between approximately one half and one order of magnitude smaller than those of canonical instanton theory. This is expected near the crossover temperature where once more the rate constants are lower than canonical instanton theory by about a factor of 2 . At low temperatures, both versions of the frequency averaged rate constants (left triangles \& crosses) deviate from canonical instanton theory (red curve) by around half an order of magnitude, however the rate constants using the finite difference approach for $d E_{b} / d \beta$ (blue, dashed curve) remain closer to those calculated using canonical instanton theory with decreasing temperatures.

It is also worth noting that while in the other test cases, eigenvalue tracing was not perceptibly better than either of the other methods in calculating $\sigma$, here it definitely outperforms both the rate constants obtained by frequency averaging and those from solving the stability matrix effectively at all temperatures. This is evidence that there are still small, non-negligible frequencies which invalidate Equation (18). Eventually however, at lower temperatures, $\beta$ will increase to such a point that Equation $(18)$ will be valid and the orange and dashed, blue curve in the upper panel of Fig. 5 will meet.

In each case studied, the rates calculated using $k_{\text {corr }}$ (dashed, red lines) of Equation (27) are very similar to those calculated using Equation (2), particularly near the crossover temperature. Below the crossover temperature, the discrepancies arise due to the methods used in determining the stability parameters, as discussed in section 2.1.1.

\section{Discussion}

The reduced instanton theory permits the calculation of chemical reaction rate constants to an accuracy which is comparable to the established instanton theory at reduced computational expense.

The method we have illustrated here involves a mixture of techniques for the calculation of both the contribution to the action due to tunnelling motion orthogonal to the instanton path and the on-the-fly calculation of the rate of change of the tunnelling energy. 
Regarding the action calculation, the stability matrix approach produces acceptable results in a temperature range near but less than the crossover temperature. In this temperature range, the tunnelling path remains short and the projection matrices between eigenvectors of neighbouring Hessians is very close to the identity, allowing easy identification of the zero stability parameters of Equation (7). In the frequency averaging approach this idealised case of projections between neighbouring Hessians is assumed fulfilled, cf. Equation (5), and is responsible for its applicability as $T \rightarrow 0 \mathrm{~K}$. In the stability matrix however, this condition must be fulfilled to within a certain, system-dependent tolerance. As long as the projections between eigenvector matrices of neighbouring Hessians is approximately 1, this method can be used with confidence. The frequency averaging approach is an inexpensive remedy when this condition can no longer be fulfilled. Another such remedy would be to rapidly increase the number of images comprising the instanton path, yet this quickly renders the reduced instanton theory less computationally efficient than canonical instanton theory.

In the range of temperatures well below the crossover temperature, the average eigenvalue approach tends to remain stable and close to the rates calculated using canonical instanton theory. In the reaction $\mathrm{H}_{2}+\mathrm{OH} \rightarrow \mathrm{H}+\mathrm{H}_{2} \mathrm{O}$ however, the presence of low-frequencies shifts the range where the average eigenvalue approach is fully applicable to much lower temperatures than either of the two previous examples. The eigenvalue tracing approach to calculating $\sigma$, as outlined elsewhere ${ }^{\sqrt{32}}$ is also useful as a cross-reference to check the validity of rate constants calculated at low temperatures if the computational effort of calculating rate constants from canonical instanton theory is prohibitive.

\section{Conclusion}

There are two main advantages over canonical instanton theory in using the reduced instanton theory. Firstly, for the calculation of rate constants near and above $T_{\mathrm{c}}$, the reduced instanton theory is superior, providing a smooth transition from temperatures below to above $T_{\mathrm{c}}$ without the spurious kink found in many Arrhenius plots created solely using canonical instanton theory. Secondly, the calculation of rate constants in canonical instanton theory requires the diagonalisation of a matrix of dimension $N P$. This diagonalisation can create a 
computational bottleneck in calculating rate constants, potentially more so than the calculation of the energies, gradients and Hessians at various coordinates especially when a fitted potential energy surface is used. In the reduced instanton theory this bottleneck is avoided, there is no need to diagonalise a matrix of dimension larger than $2 N$.

An intermediate approach is to use canonical instanton theory with the correction factor of Equation (27). It provides rate constants at the accuracy and reliability of canonical instanton theory while alleviating the overestimation of rate constants close to, but below $T_{\mathrm{c}}$.

We have also identified certain pitfalls in the theory, such as the necessity of the frequency averaging approach for calculating $\sigma$ as an insurance against the eventual failure of the stability matrix when calculating rate constants at lower temperatures. There may be some utility in representing the instanton as a series of periodic basis functions ${ }^{3}$ in order to continue locating the zero eigenvalues in the stability matrix eigenvalue spectrum at low temperatures, we are currently exploring implementing such a solution in our next program version.

Also implemented is a simple method to approximate the rate of change in the tunnelling energy with temperature. Since the formula is analytic, there is no limit to its precision, though as shown best in the reaction $\mathrm{H}_{2}+\mathrm{OH} \rightarrow \mathrm{H}+\mathrm{H}_{2} \mathrm{O}$ precision is not a guarantee of accuracy. Fortunately, any deviation in $d E_{b} / d \beta$ is damped since it enters the rate equation under the square root. Despite the seemingly large difference in $d E_{b} / d \beta$ calculated by each method for $\mathrm{H}_{2}+\mathrm{OH} \rightarrow \mathrm{H}+\mathrm{H}_{2} \mathrm{O}$ (cf. Fig. 1) the actual effect on rate constants, at temperatures ranging from near $T_{c}$ to moderately low, is only about half an order of magnitude. Clearly though at very low temperatures, this deviation increases, hence improving our simple approximation to $d E_{b} / d \beta$ is one of the tasks for our group going forward.

Finally, it is prudent to use canonical instanton theory in conjunction with the reduced theory in order to benchmark rate constants in the very low temperature range. It is recommended that when calculating rate constants using the reduced theory that, at the lowest temperature of interest, one should perform a single calculation in the canonical instanton theory. In doing so, one can visualise the results from using either the stability matrix, or the frequency averaging approach. Though it is certain that both approaches cannot calculate accurate rate constants at arbitrarily low temperatures, the point at which their results begin 
to strongly deviate from each other is entirely system dependent. In this regard, one final

calculation using canonical instanton theory may help in deciding which of the two methods is the more reliable in the temperature regime of interest and should not add significantly to the total computational expenditure.

\section{ACKNOWLEDGMENTS}

This work was financially supported by the European Union's Horizon 2020 research and innovation programme (grant agreement No. 646717, TUNNELCHEM).

\section{References}

1. J. Kästner, WIREs Comput. Mol. Sci. 4, 158 (2014).

2. J. Meisner and J. Kästner, Angew. Chem. Int. Ed. 55, 5400 (2016).

3. M. Kryvohuz, J. Chem. Phys. 134, 114103 (2011).

4. M. Kryvohuz and R. A. Marcus, J. Chem. Phys. 137, 134107 (2012).

5. M. Kryvohuz, J. Chem. Phys. 138, 244114 (2013).

6. J. S. Langer, Ann. Phys. (N.Y.) 41, 108 (1967).

7. J. S. Langer, Ann. Phys. (N.Y.) 54, 258 (1969).

8. W. H. Miller, J. Chem. Phys. 62, 1899 (1975).

9. C. G. Callan Jr. and S. Coleman, Phys. Rev. D 16, 1762 (1977).

10. S. Coleman, Phys. Rev. D 15, 2929 (1977).

11. E. Gildener and A. Patrascioiu, Phys. Rev. D 16, 423 (1977).

12. I. Affleck, Phys. Rev. Lett. 46, 388 (1981).

13. S. Coleman, Nucl. Phys. B 298, 178 (1988). 
14. G. A. Voth, D. Chandler, and W. H. Miller, J. Chem. Phys. 91, 7749 (1989).

15. P. Hänggi, P. Talkner, and M. Borkovec, Rev. Mod. Phys. 62, 251 (1990).

16. V. Benderskii and D. Makarov, Phys. Lett. A 161, 535 (1992).

17. J. Cao and G. A. Voth, J. Chem. Phys. 105, 6856 (1996).

18. W. Siebrand, Z. Smedarchina, M. Z. Zgierski, and A. Fernández-Ramos, Int. Rev. Phys. Chem. 18, 5 (1999).

19. J. O. Richardson and S. C. Althorpe, J. Chem. Phys. 131, 214106 (2009).

20. T. P. M. Goumans and J. Kästner, Angew. Chem. Int. Ed. 49, 7350 (2010).

21. S. C. Althorpe, J. Chem. Phys. 134, 114104 (2011).

22. J. B. Rommel and J. Kästner, J. Chem. Phys. 134, 184107 (2011).

23. J. Kästner, Chem. Eur. J. 19, 8207 (2013).

24. G. Gamow, Z. Phys. 51, 204 (1928).

25. B. M. Garraway and K.-A. Suominen, Rep. Prog. Phys. 58, 365 (1995).

26. S. Andersson, G. Nyman, A. Arnaldsson, U. Manthe, and H. Jónsson, J. Phys. Chem. A 113, 4468 (2009).

27. R. Pérez de Tudela, Y. V. Suleimanov, J. O. Richardson, V. Sez Rbanos, W. H. Green, and F. J. Aoiz, J. Phys. Chem. Lett. 5, 4219 (2014).

28. A. Arnaldsson, Ph.D. thesis, University of Washington, University of Washington Graduate School (2007).

29. T. P. M. Goumans and J. Kästner, J. Phys. Chem. A 115, 10767 (2011).

30. J. O. Richardson, J. Chem. Phys. 144, 114106 (2016).

31. J. O. Richardson, Farad. Discuss. 195, 49 (2016). 
32. S. R. McConnell, A. Löhle, and J. Kästner, J. Chem. Phys. 146, 074105 (2017).

33. H. Kleinert, Path Integrals in Quantum Mechanics, Statistics, Polymer Physics, and Financial Markets (World Scientific, Singapore, 2009), 5th ed.

34. J. Kästner, J. M. Carr, T. W. Keal, W. Thiel, A. Wander, and P. Sherwood, J. Phys. Chem. A 113, 11856 (2009).

35. J. B. Rommel, T. P. M. Goumans, and J. Kästner, J. Chem. Theory Comput. 7, 690 (2011).

36. M. C. Gutzwiller, J. Math. Phys. 12, 343 (1971).

37. T. Schwidder, Ph.D. thesis, University of Stuttgart. (2013).

38. I. M. Gel'fand and A. M. Yaglom, J. Math. Phys. 1, 48 (1960).

39. S. Álvarez-Barcia, J. R. Flores, and J. Kästner, J. Phys. Chem. A 118, 78 (2014).

40. T. Lamberts, P. K. Samanta, A. Köhn, and J. Kästner, Phys. Chem. Chem. Phys. 18, 33021 (2016).

41. L. Song and J. Kästner, Phys. Chem. Chem. Phys. 18, 29278 (2016).

42. S. Álvarez-Barcia, M.-S. Russ, J. Meisner, and J. Kästner, Faraday Disc. 195, 69 (2016).

43. J. Meisner and J. Kästner, J. Chem. Phys. 144, 174303 (2016).

44. T. Lamberts, G. Fedoseev, J. Kästner, S. Ioppolo, and H. Linnartz, Astron. Astrophys. 599, A132 (2017).

45. H. Kobayashi, H. Hidaka, T. Lamberts, T. Hama, H. Kawakita, J. Kästner, and N. Watanabe, Astrophys. J. 837, 155 (2017).

46. C. Eckart, Phys. Rev. 35, 1303 (1930).

47. R. P. Bell, Trans. Faraday Soc. 55, 1 (1959).

48. S. Chapman, B. C. Garrett, and W. H. Miller, J. Chem. Phys. 63, 2710 (1975). 
49. G. Mills and H. Jónsson, Phys. Rev. Lett. 72, 1124 (1994).

50. G. Mills, H. Jónsson, and G. K. Schenter, Surf. Sci. 324, 305 (1995).

51. G. Mills, G. K. Schenter, D. E. Makarov, and H. Jónsson, Chem. Phys. Lett. 278, 91 (1997).

52. Z. Smedarchina, W. Siebrand, A. Fernández-Ramos, and Q. Cui, J. Am. Chem. Soc. 125, $243(2003)$.

53. T. Qian, W. Ren, J. Shi, W. E, and P. Sheng, Physica A 379, 491 (2007).

54. T. P. M. Goumans and S. Andersson, Mon. Not. R. Astron. Soc. 406, 2213 (2010).

55. T. P. M. Goumans, Mon. Not. R. Astron. Soc. 415, 3129 (2011).

56. T. P. M. Goumans, Mon. Not. R. Astron. Soc. 413, 2615 (2011).

57. H. Jónsson, Proc. Nat. Acad. Sci. U.S.A. 108, 944 (2010).

58. J. Meisner, J. B. Rommel, and J. Kästner, J. Comput. Chem. 32, 3456 (2011).

59. D. M. Einarsdóttir, A. Arnaldsson, F. Óskarsson, and H. Jónsson, Lect. Notes Comput. Sci. 7134, 45 (2012).

60. J. B. Rommel, Y. Liu, H.-J. Werner, and J. Kästner, J. Phys. Chem. B 116, 13682 (2012).

61. M. Kryvohuz, J. Phys. Chem. A 118, 535 (2014).

62. K. Müller and L. D. Brown, Theor. Chim. Acta 53, 75 (1979).

63. P. R. Schreiner, H. P. Reisenauer, D. Ley, D. Gerbig, C.-H. Wu, and W. D. Allen, Science 332, 1300 (2011).

64. D. Ley, D. Gerbig, and P. R. Schreiner, Org. Biomol. Chem. 10, 3781 (2012).

65. J. Chen, X. Xu, X. Xu, and D. H. Zhang, J. Chem. Phys. 138, 154301 (2013). 